\title{
Fast thermalization and Helmholtz oscillations of an ultracold Bose gas
}

\author{
D.J. Papoular ${ }^{1}$, L.P. Pitaevskii ${ }^{1,2}$, S. Stringari ${ }^{1}$ \\ ${ }^{1}$ INO-CNR BEC Center and Dipartimento di Fisica, Università di Trento, 38123 Povo, Italy and \\ ${ }^{2}$ Kapitza Institute for Physical Problems, Kosygina 2, 119334 Moscow, Russia
}

(Dated: January 18, 2020)

\begin{abstract}
We analyze theoretically the transport properties of a weakly-interacting ultracold Bose gas enclosed in two reservoirs connected by a constriction. We assume that the transport of the superfluid part is hydrodynamic, and we describe the ballistic transport of the normal part using the LandauerBüttiker formalism. Modeling the coupled evolution of the phase, atom number, and temperature mismatches between the reservoirs, we predict that Helmholtz (plasma) oscillations, induced by an initial imbalance in atom numbers, can be observed at non-zero temperatures below $T_{c}$. We show that, because of its strong compressibility, the ultracold Bose gas is characterized by a fast thermalization compared to the damping time for plasma oscillations, accompanied by a fast transfer of the normal component through the constriction. This fast thermalization also affects the gas above $T_{c}$, where we present an explicit comparison to the ideal fermionic case.
\end{abstract}

PACS numbers: 47.37.+q, 67.10.Jn, 03.75.Kk

Transport without friction is a signature property of superfluidity, spectacularly illustrated in the fountain effect of liquid Helium [1]. Its observation relies on the use of a superleak, which lets the superfluid through while blocking the normal part. Superleaks are familiar elements in the context of experiments on liquid helium [2], but their design in the context of ultracold gases remains an open question. Their implementation would allow, for instance, the implementation of new adiabatic cooling schemes [3], the efficient excitation of second sound [2], and, more generally, an advanced control over transport phenomena.

Recent experiments have initiated the exploration of the transport properties of ultracold atomic gases $[4-10]$ in geometries comprising two reservoirs separated by a potential barrier or by a constriction (see Fig. 11). The constriction-based geometry is related to those investigated in mesoscopic physics [11]. It has already been used to demonstrate the concept of contact resistance [7], to investigate superflow [8], and to observe thermoelectric effects [9, 12] in ultracold Fermi gases. Superfluids trapped within two connected reservoirs are expected to undergo plasma oscillations, which are analogous to the oscillations of a gas in between two connected Helmholtz resonators [13, §69]. These oscillations have been extensively studied in the context of liquid helium [14]. Similar oscillations have also been observed with ultracold Bose gases in double-well potentials [15, 16].

In ultracold Fermi gases, the occurrence of BCStype superfluidity occurs at reasonably high temperatures only in the presence of strong interactions [17]. In this case, both the superfluid and normal parts of the quantum fluid are deep in the hydrodynamic regime, which affords a strong analogy with superfluid Helium [18]. However, it also makes it more difficult to tell the behavior of the superfluid fraction apart from that of the normal fraction. Hence, in the present Letter, we focus on weakly-interacting bosonic gases, where the parameters

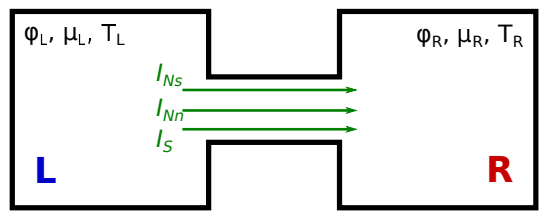

FIG. 1. Two reservoirs can exchange particles and heat through a constriction.

can be chosen such that superfluid transport is hydrodynamic whereas normal transport through the channel is ballistic.

We develop a theory describing the transport properties of weakly-interacting uniform Bose gases under these conditions, reflecting the different transport regimes for the superfluid and normal parts. We use it to show that plasma oscillations are observable even at non-zero temperatures below $T_{c}$, and provide a first description of the damping mechanism due to the coupling between the superfluid and normal parts. We also show that the large compressibility of the Bose gas leads to surprisingly fast thermalization compared to the damping time of the transport phenomena. Below $T_{c}$, this causes an efficient transport of the normal part at short times; above $T_{c}$, it yields a key difference compared to ideal fermionic gases.

We describe the ballistic transport of the normal part of the fluid using the Landauer-Büttiker formalism for quantum transport [19, chap. 2]. To our knowledge, the present work is the first application of this formalism to massive bosons. It had previously been applied to (massless) phonons to determine the quantum properties of heat conductance [20].

We assume that the two compartments of Fig. 11 are box traps with the same volume $V^{L}=V^{R}$, each enclosing a uniform superfluid. We model the constriction by an isotropic radial harmonic trap of frequency $\omega_{\perp} / 2 \pi$. The hydrodynamic assumption for superfluid 
transport through the constriction [21, chap. 5] is valid if $\hbar \omega_{\perp} \ll g n$, where $n$ is the mean gas density inside the constriction, $g=4 \pi \hbar^{2} a / m$ is the interaction constant, $a$ is the scattering length and $m$ is the atomic mass.

We call $\delta N_{s}=N_{s}^{R}-N_{s}^{L}$ and $\delta N_{n}=N_{n}^{R}-N_{n}^{L}$ the difference in superfluid and normal atom numbers between the right and left compartments of Fig. 1 and $\delta S=S^{R}-S^{L}$ the analogous entropy difference. We focus on small deviations from the homogeneous situation. In this linear-response regime, the superfluid current $I_{N_{s}}$, the normal current $I_{N_{n}}$, and the entropy current $I_{S}$, corresponding to the three differences above, are linear functions of the small differences in phase $\delta \phi$, chemical potential $\delta \mu$, and temperature $\delta T$ between the two reservoirs, which we write in matrix form as:

$$
\left(\begin{array}{c}
I_{N_{s}} \\
I_{N_{n}} \\
I_{S} / k_{B}
\end{array}\right)=\left(\begin{array}{ccc}
2 I_{J} & 0 & 0 \\
0 & L_{11} & L_{12} \\
0 & L_{12} & L_{22}
\end{array}\right)\left(\begin{array}{c}
\hbar \delta \phi \\
\delta \mu \\
k_{B} \delta T
\end{array}\right) .
$$

The first line in the matrix reflects the definition of the superfluid current, $\mathbf{j}_{s}=n_{s} \mathbf{v}_{s}$, where $n_{s}$ is the mean superfluid density in the reservoirs and $\mathbf{v}_{s}$ is the superfluid velocity. The latter satisfies $m \mathbf{v}_{s}=\hbar \nabla \phi$, with $m$ being the atomic mass. For the geometry of Fig. 1, we find $I_{J}=n_{s} A / m l$, where $l$ is the constriction length and $A=\pi g n_{s} / m \omega_{\perp}^{2}$ is its effective Thomas-Fermi section. The two zeroes in the first column reflect the fact that the normal-part quantities $\delta N_{n}$ and $\delta S$ do not explicitly depend on the superfluid phase difference $\delta \phi$. The coefficients $\left(L_{i j}\right)$ describe the ballistic transport of the normal part and the entropy. Assuming that $k_{B} T \gg g n$, the excitations exchanged by the reservoirs are particles, and an analysis of the role of interactions using HartreeFock theory reveals that the ideal-gas expressions for the $L_{i j}$ 's are applicable. This assumption on $T$ rules out low-temperature collective phenomena, such as anomalous phonon transmission 22] or Andreev reflection 23]. For uniform Bose gases, this condition is easy to satisfy while maintaining the presence of superfluid $\left(T<T_{c}\right)$, because the ratio $g n / k_{B} T_{c}$ is of the order of 0.04 .

We calculate the $L_{i j}$ 's using the Landauer-Büttiker formalism [19, chap. 2]. We describe the excitations in each reservoir using Bose distribution functions $\eta^{B}$, whose difference $\delta \eta^{B}=\eta_{R}^{B}-\eta_{L}^{B}$ satisfies $\delta \eta^{B}=$ $\partial \eta^{B} /\left.\partial \mu\right|_{T} \delta \mu+\partial \eta^{B} /\left.\partial T\right|_{\mu} \delta T$. For temperatures below $T_{c}$, the $L_{i j}$ 's are given by:

$$
\begin{aligned}
& h L_{11}=-\frac{\pi^{2}}{6}\left(\frac{k_{B} T}{\hbar \omega_{\perp}}\right)^{2}, \\
& L_{12}=L_{21}=\frac{18}{\pi^{2}} \zeta(3) L_{11}, \quad L_{22}=\frac{4 \pi^{2}}{5} L_{11} .
\end{aligned}
$$

The $L_{i j}$ 's do not depend on the constriction length $(\sim 5 \mu \mathrm{m})$, because it is much shorter than the thermal mean free path inside the reservoirs $(\sim 100 \mu \mathrm{m})$. Furthermore, the $L_{i j}$ 's all share the same dependence on $T$

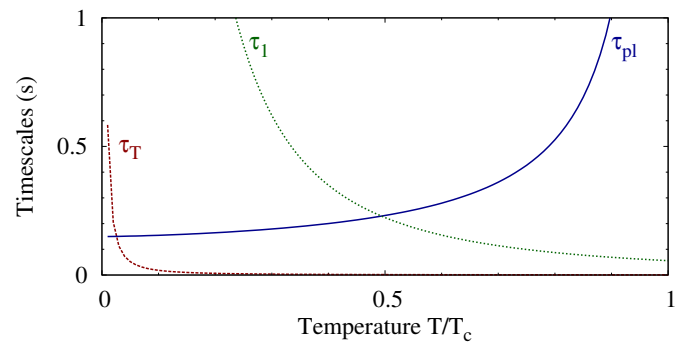

FIG. 2. The three timescales $\tau_{T}$ (dashed red), $\tau_{\mathrm{pl}}$ (solid blue), $\tau_{1}$ (dotted green) defining the transport of an ultracold ${ }^{87} \mathrm{Rb}$ Bose gas. Calculated for $N=10^{5}$ atoms in each reservoir, density $n=10^{19}$ atoms $/ \mathrm{m}^{3}$, constriction frequency $\omega_{\perp} / 2 \pi=$ $15 \mathrm{~Hz}$ and constriction length $l=5 \mu \mathrm{m}$.

and $\omega_{\perp}$. This second property is an important difference with respect to the fermionic case [9], where the chemical potential is of the order of the Fermi energy and, hence, enters in the expression for the transport coefficients.

The coupling between the superfluid and normal parts arises from the equation of state, which involves the total gas density in each reservoir, e.g. $n^{R}=\left(N_{s}^{R}+N_{n}^{R}\right) / V^{R}$, and from the equation dictating the evolution of the superfluid velocity, $\hbar \partial_{t} \delta \phi=-\delta \mu[21]$. Combining these equations with the currents given by Eq. (1), we obtain a differential system describing the evolution of $\delta \phi, \delta N$, and $\delta T[24]$ :

$$
\tau_{1} \frac{d}{d t}\left(\begin{array}{c}
\frac{\hbar \delta \phi}{\tau_{1}} \\
\frac{\delta N}{\kappa_{T}} \\
k_{B} \delta T
\end{array}\right)=\left(\begin{array}{ccc}
0 & -1 & 0 \\
\left(\omega_{\mathrm{p} 1} \tau_{1}\right)^{2} & -1 & +\mathcal{S} \\
0 & \mathcal{S} / \ell & -\tau_{1} / \tau_{T}
\end{array}\right)\left(\begin{array}{c}
\frac{\hbar \delta \phi}{\tau_{1}} \\
\frac{\delta N}{\kappa_{T}} \\
k_{B} \delta T
\end{array}\right) .
$$

In Eq. (3), $\kappa_{T}=\partial N /\left.\partial \mu\right|_{T}$ is the isothermal compressibility, $C_{N}=T \partial S /\left.\partial T\right|_{N}$ is the heat capacity, $\ell=C_{N} / \kappa T$ is their ratio, and the Seebeck coefficient $\mathcal{S}=-\partial \mu /\left.\partial T\right|_{N}-$ $L_{12} / L_{11}$ encodes the thermoelectric properties of the gas. Equation (3) introduces three timescales:

$$
\tau_{1}=\frac{\kappa}{-L_{11}}, \quad \tau_{\mathrm{pl}}=2 \pi \sqrt{\frac{\kappa}{2 I_{J}}}, \quad \tau_{T}=\frac{C_{N} / T}{-L_{22}},
$$

where $\tau_{1}$ is a damping time associated with normal transport, the bare plasma period $\tau_{\mathrm{pl}}=2 \pi / \omega_{\mathrm{pl}}$ is associated to superfluid transport, and $\tau_{T}$ is the thermalization time. The time $\tau_{1}$ determines the damping of plasma oscillations and thermolectric effects.

Weakly-interacting Bose gases are characterized by a very large compressibility $\left(\kappa_{T}=N / g n\right.$ for $\left.T<T_{c}\right)$, whereas $C_{N} / N k_{B}$ remains finite (see Fig. 3 left). Hence, the ratio $\ell$ is very small, of the order of a few $10^{-2}$, which is a key difference with respect to both ideal Fermi gases $(\ell \sim 1$, Fig. 3 center $)$ and liquid Helium $4(\ell \sim 10$ close to the superfluid transition, Fig. 3right). This specific property of Bose gases leads to $\tau_{T} \ll \tau_{1}$, i.e. thermalization is much faster than the damping due to normal transport, 

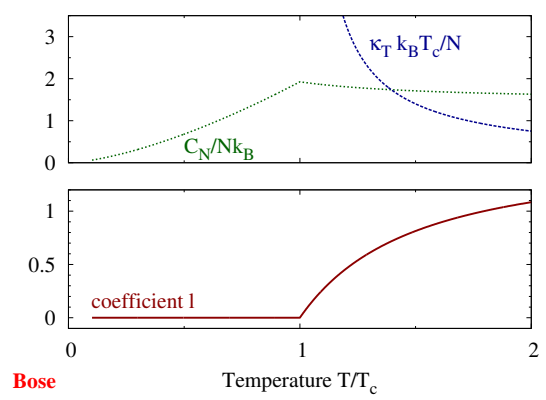
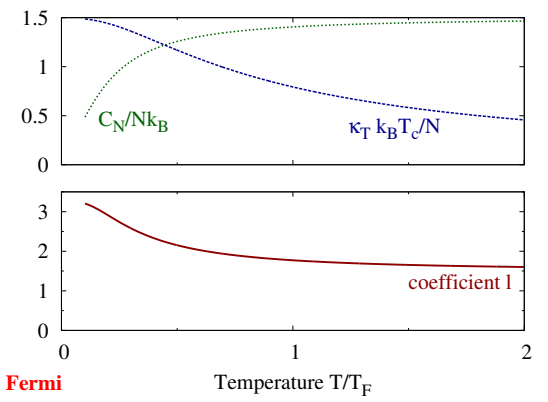

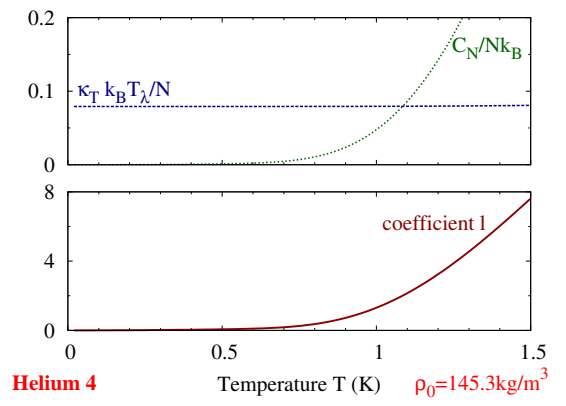

FIG. 3. Specific heat $C_{N}$ (dotted green), compressibility $\kappa_{T}$ (dashed blue), and their ratio $\ell$ (solid red), for ideal Bose (left) and Fermi (center) gases, and for liquid helium 4 (right, density $\rho_{0}=145.3 \mathrm{~kg} / \mathrm{m}^{3}$, calculated using the thermodynamic data in [25]).

as illustrated on Fig. 2, Furthermore, for temperatures high enough for Hartree-Fock theory to hold $\left(k_{B} T \gtrsim\right.$ a few $g n)$, but low enough for the superfluid fraction $N_{s} / N=1-\left(T / T_{c}\right)^{3 / 2}$ to be substantial $\left(T / T_{c} \lesssim 0.5\right)$, the three timescales satisfy $\tau_{T} \ll \tau_{\mathrm{pl}}<\tau_{1}$. On the other hand, if $T \gtrsim T_{c}$, the superfluid is absent and our description reduces to the dynamical system on $\delta N$ and $\delta T$ introduced in [9], which corresponds to the lower right $2 \times 2$ block of the matrix in Eq. (3).

Plasma oscillations. We now turn to the analysis of plasma oscillations in the geometry of Fig. 1. These oscillations can be excited by introducing an initial atom number mismatch $\delta N$ between the two reservoirs. We have predicted their occurrence at $T=0$ by numerically solving the Gross-Pitaevskii equation, using a CrankNicolson scheme [26, 27]. We have investigated a 2D geometry inspired by [28], as well as the cylindrically symmetric three-dimensional geometry corresponding to Fig. [1. Our results validate the hydrodynamic approach for superfluid transport and yield promising orders of magnitude for the plasma oscillation frequency $\omega_{\mathrm{pl}}^{(0)}$, of the order of a few $\mathrm{Hz}$.

Our model allows us to investigate plasma oscillations at non-zero temperatures. First, our Hartree-Fock description shows that the bare plasma frequency $\omega_{\mathrm{pl}}$ scales with $a / l^{1 / 2}$, whereas the damping factor $\omega_{\mathrm{pl}} \tau_{1}$ is proportional to $\left(T_{c} / T\right)^{2} / \sqrt{l}$ and does not depend on $a$. Therefore, the observation of oscillations will be favored by using smaller constriction lengths, lower temperatures $T / T_{c}$, and larger scattering lengths $a$. Plasma oscillations occur if the matrix entering Eq. (3) has two complex-conjugate eigenvalues with negative real parts, $\left(-1 / \tau_{\text {damp }} \pm i \omega_{\text {osc }}\right)$. In this case, the plasma oscillation frequency is $\omega_{\text {osc }} / 2 \pi$ and the damping time is $\tau_{\text {damp }}$. Figure 4 left shows the temperature dependence of $\omega_{\text {osc }}$ and $\tau_{\text {damp }}$ for a typical ultracold ${ }^{87} \mathrm{Rb}$ Bose gas below $T_{c}$. Oscillations occur for temperatures up to $0.95 T_{c}$. For higher temperatures, the superfluid fraction becomes negligible, and the damping time coincides with that predicted by the normal-part model of Ref. [9].

Thermalization being a fast process compared to the timescales $\tau_{\mathrm{pl}}$ and $\tau_{1}$ causes the evolution of $\delta T$ to approximately decouple from that of $\delta \phi$ and $\delta N$. Hence, the dynamics of these latter two quantities is almost isothermal and is piloted by the upper left $2 \times 2$ block of the matrix entering Eq. (3). The maximum amplitude of the temperature oscillations can be determined by assuming that the dynamics of $\delta T$ is driven by that of $\delta N$ and $\delta \phi$ :

$$
\frac{\delta T_{\max }}{T}=\frac{g n}{k_{B} T} \frac{\mathcal{S}}{\mathcal{S}^{2}+L} \frac{\delta N_{0}}{N} .
$$

The presence of the factor $g n / k_{B} T$ in Eq. (5) causes $\delta T_{\max } / T$ to remain small and confirms the nearisothermal nature of these oscillations. Figure 4 left shows that the oscillation frequency and damping time predicted by the isothermal model (green) are in good agreement with the full calculation (red). Hence, the main decay mechanism is due to the presence of the normal part. Thermoelectric effects, neglected in the isothermal model, are seen to affect mostly the damping time, causing it to lengthen.

The plasma oscillations caused by an initial number mismatch $\delta N_{0} / N=0.1$ are shown on Fig. 4 center, for the same parameters as those used in Fig. 22 This figure also shows the number of normal atoms that have traveled through the constriction, $\delta N_{n}^{\operatorname{tr}}(t)$ [29], to reveal that the plasma oscillations are performed almost exclusively by the superfluid part.

Thermalization at temperatures below $T_{c}$. In order to reveal the key role played by fast thermalization in ultracold Bose gases, we now consider the response of the system to an initial temperature mismatch $\delta T_{0}$. We consider temperatures $T / T_{c} \lesssim 0.5$. In this case, the dynamics of the system at small times of the order of $\tau_{T}$ is driven by the relaxation of temperature towards $\delta T=0$. This fast process quickly converts the initial temperature mismatch $\delta T_{0}$ into a number imbalance $\delta N_{\max }$ :

$$
\frac{\delta N_{\max }}{N}=\frac{15}{4} \frac{\zeta(5 / 2)}{\zeta(3 / 2)} \frac{\mathcal{S}}{\mathcal{S}^{2}+L}\left(\frac{T}{T_{c}}\right)^{3 / 2} \frac{\delta T_{0}}{T} .
$$

The sign of $\delta N_{\max }$ is dictated by the Seebeck coefficient $\mathcal{S}$, which is negative, just like for fermions [9]. 

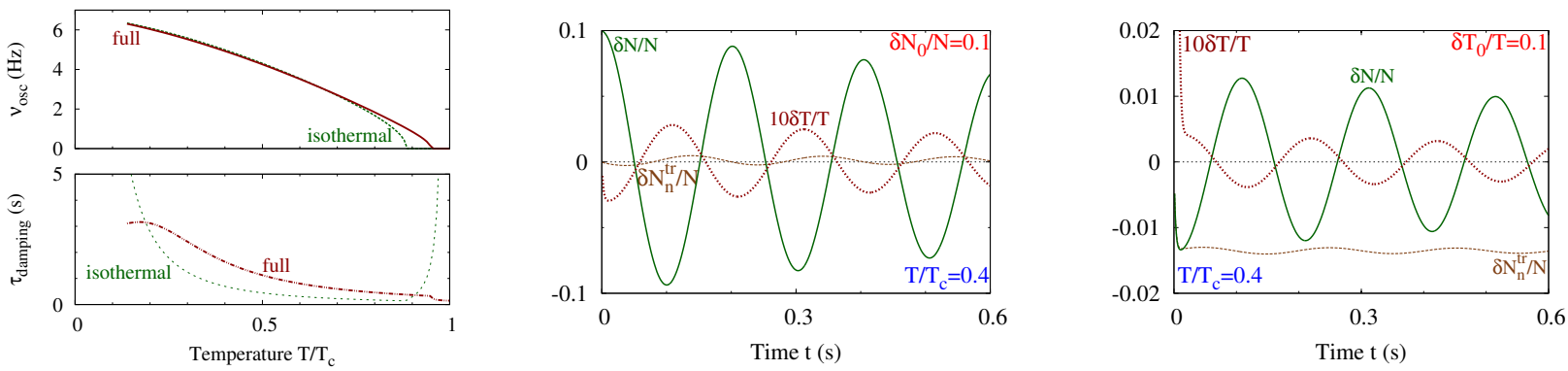

FIG. 4. Superfluid oscillations in an ultracold ${ }^{87} \mathrm{Rb}$ Bose gas, for the parameters used in Fig. 2. Left: frequency $\nu_{\mathrm{osc}}=\omega_{\mathrm{osc}} / 2 \pi$ (top) and damping time $\tau_{\text {damp }}$ (bottom) for plasma oscillations at non-zero temperatures below $T_{c}$, calculated using Eq. 3 (red "full") and its isothermal limit (dashed green "isothermal"). Center: the initial imbalance in atom numbers $\delta N_{0} / N=0.1$ causes quasi-isothermal oscillations. Right: the initial temperature mismatch $\delta T_{0} / T=0.1$ yields fast thermalization accompanied by an efficient transport of the thermal part at short times, followed by quasi-isothermal oscillations. In both cases, $T / T_{c}=0.4$, and we plot the time evolution of the differences in atom numbers $\delta N / N$ (solid green), temperature $\delta T / T$ (dotted red, multiplied by 10), and transported thermal part atoms $\delta N_{n}^{\text {tr }} / N$ (dashed brown).

Furthermore, according to Eq. (1), temperature variations do not directly couple to the motion of the superfluid part. Hence, this fast relaxation process almost exclusively drives the transport of normal atoms. On a longer timescale, the oscillation then proceeds quasiisothermally as before, with the frequency $\omega_{\text {osc }}$ and the damping time $\tau_{\text {damp }}$. This process is illustrated on Fig. 4 right, for $\delta T_{0} / T=0.1$ and the parameters used in Fig. 2.

Thermalization at temperatures above $T_{c}$. In Bose gases, the ratio $\ell$ remains small for temperatures $T \gtrsim T_{c}$, where the physics is captured by the ideal-gas model and a direct comparison with fermions is possible (see Fig. 3 left and center). The gas contains no superfluid part, and the dynamics of $\delta N$ and $\delta T$ are described by the lower right $2 \times 2$ block of Eq. (3), which coincides with the model of Ref. [9]. Equation (44) shows that the thermalization time $\tau_{T}$ is determined by the specific heat, which is of the same order of magnitude for Bose and Fermi gases. However, the damping time $\tau_{1}$ involves the compressibility, which is much larger for bosons than for fermions. Therefore, damping is much slower in Bose gases than in Fermi gases. The variation of $\delta N$ reflects the two timescales $\tau_{T}$ and $\tau_{T}$. In both cases, the Seebeck coefficient $\mathcal{S}$ is negative, therefore $\delta N$ first decreases towards negative values. It reaches a minimum for short times $t_{m} \simeq \tau_{T} \ln \left(\tau_{1} / \tau_{T}\right)$, whose value $\delta N_{m}=\delta T_{0} / T C_{N} \mathcal{S} /\left(\mathcal{S}^{2}+\ell\right)$ does not depend critically on the statistics. However, the difference between bosons and fermions is apparent during the long-time relaxation towards $\delta N=0$. Figure 5 compares the cases of bosonic ${ }^{41} \mathrm{~K}$ at the temperature $T / T_{c}=1.1$ and fermionic ${ }^{40} \mathrm{~K}$ at the temperature $T / T_{F}=1.1$, with $T_{F}$ being the Fermi energy. These two isotopes differ only by the statistics which they obey, and the relaxation is 50 times longer for bosons $\left(\tau_{1}^{B} \sim 1.5 \mathrm{~s}\right)$ than for fermions $\left(\tau_{1}^{F} \sim 30 \mathrm{~ms}\right)$.

Strictly speaking, the plasma oscillations we have analyzed for $T<T_{c}$ are not Josephson oscillations. These would occur for $\mu \ll \hbar \omega_{\perp}$, which is opposite to our hy-

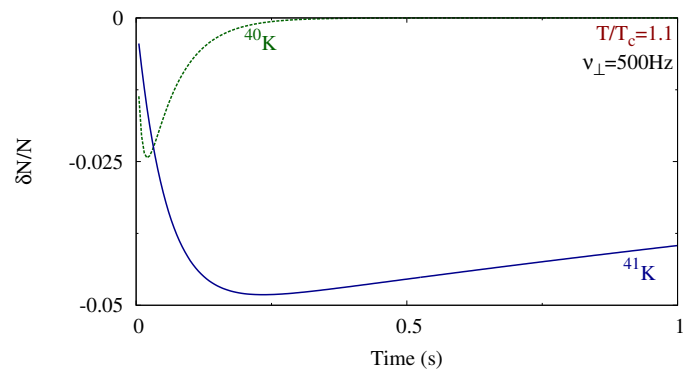

FIG. 5. Evolution of $\delta N / N$ following an initial temperature imbalance $\delta T_{0} / T=0.1$ for bosonic ${ }^{41} \mathrm{~K}$ (solid blue, $T=1.1 T_{c}$ ) and fermionic ${ }^{40} \mathrm{~K}$ (dashed green, $T=1.1 T_{F}$ ). No superfluid is present, and the constriction is more stringent $\left(\omega_{\perp} / 2 \pi=\right.$ $500 \mathrm{~Hz}$ ) to achieve bosonic decay times of the order of $1 \mathrm{~s}$.

drodynamicity condition for the superfluid flow. However, in the linear-response limit we envisage, the two equations determining the superfluid dynamics at $T=0$ are formally equivalent to the Josephson equations 21, chap. 15]. A qualitative difference with true Josephson oscillations will emerge in the non-linear regime, where deviations from the law $\Delta N_{s}=2 I_{J} \sin (\Delta \phi)$ should be seen. The classical-to-quantum crossover to Josephson oscillations can be explored numerically at $T=0$ by varying the constriction geometry.

We have shown that, in the case where the transport of the normal part through the constriction is ballistic, plasma oscillations can be observed at non-zero temperatures below $T_{c}$, and that thermalization between the reservoirs is fast compared to the oscillation period, causing an efficient transport of the normal part at short times (see Fig. 4 right). A possible way to inhibit normal transport, and thus to realize a superleak, is to add a disordered potential inside the constriction, for instance by projecting a speckle [9], in analogy with the fine powders used in the historical superleaks [1]. The presence of disorder should not impede superfluid flow [30]. The 
anomalously fast thermalization will disappear if $L_{22}$ is chosen sufficiently small to achieve $\tau_{T}>\tau_{\mathrm{pl}}$.

We are grateful to S. Balibar, I. Carusotto, G. Ferrari, and A. Georges for fruitful discussions. This work has been supported by ERC through the QGBE grant.

[1] J. F. Allen and H. Jones, Nature 141, 244 (1938).

[2] R. J. Donnelly, Phys. Today 62 (2009).

[3] D. J. Papoular, G. Ferrari, L. P. Pitaevskii, and S. Stringari, Phys. Rev. Lett. 109, 084501 (2012).

[4] E. L. Hazlett, L.-C. Ha, and C. Chin, arXiv:1306.4018 (2013).

[5] A. Rancon, C. Chin, and K. Levin, arXiv:1311.0769 (2013).

[6] F. Jendrzejewski, S. Eckel, N. Murray, C. Lanier, M. Edwards, C. J. Lobb, and G. K. Campbell, arXiv:1402.3335 (2014).

[7] J.-P. Brantut, J. Meineke, D. Stadler, S. Krinner, and T. Esslinger, Science 337, 1069 (2012).

[8] D. Stadler, S. Krinner, J. P. Brantut, and T. Esslinger, Nature 491, 736 (2012).

[9] J. P. Brantut, C. Grenier, J. Meineke, D. Stadler, S. Krinner, C. Kollath, T. Esslinger, and A. Georges, Science 342, 713 (2013).

[10] J. G. Lee, B. J. McIlvain, C. J. Lobb, and W. T. Hill, III, Sci. Rep. 3 (2013).

[11] Y. V. Nazarov, Quantum Transport: Introduction to Nanoscience (Cambridge University Press, 2009).

[12] C. Grenier, C. Kollath, and A. Georges, arXiv:1209.3942 (2012).

[13] L. D. Landau and E. M. Lifshitz, Fluid Mechanics (Pergamon, 1987).

[14] Y. Sato and R. E. Packard, Rep. Prog. Phys. 75, 016401 (2012).

[15] M. Albiez, R. Gati, J. Fölling, S. Hunsmann, M. Cristiani, and M. K. Oberthaler, Phys. Rev. Lett. 95, 010402
$(2005)$

[16] L. J. LeBlanc, A. B. Bardon, J. McKeever, M. H. T. Extavour, D. Jervis, J. H. Thywissen, F. Piazza, and A. Smerzi, Phys. Rev. Lett. 106, 025302 (2011).

[17] S. Giorgini, L. P. Pitaevskii, and S. Stringari, Rev. Mod. Phys. 80, 1215 (2008).

[18] L. A. Sidorenkov, M. K. Tey, R. Grimm, Y.-H. Hou, L. P. Pitaevskii, and S. Stringari, Nature 498, 78 (2013).

[19] S. Datta, Electronic Transport in Mesoscopic Systems (Cambridge University Press, 1995).

[20] K. Schwab, E. A. Henriksen, J. M. Worlock, and M. L. Roukes, Nature 404, 974 (2000).

[21] L. P. Pitaevskii and S. Stringari, Bose-Einstein condensation (Oxford Clarendon Press, 2003).

[22] Y. Kagan, D. L. Kovrizhin, and L. A. Maksimov, Phys. Rev. Lett. 90, 130402 (2003).

[23] I. Zapata and F. Sols, Phys. Rev. Lett. 102, 180405 (2009).

[24] In Eq. (3), we have set to 0 the top right and lower left matrix coefficients. These coefficients are respectively equal to $\alpha / \kappa$ and $-\left(\omega_{\mathrm{pl}} \tau_{1}\right)^{2} \alpha /(\kappa \ell)$, where $\alpha=\partial N /\left.\partial T\right|_{\mu}$, and they have a negligible impact on the dynamics of the system. We have retained them in our numerical calculations.

[25] V. D. Arp, Int. J. Thermophys. 26, 1477 (2005).

[26] W. H. Press, S. A. Teukolsky, W. T. Vetterling, and B. P. Flannery, Numerical Recipes, 3rd ed. (Cambridge University Press, 2007).

[27] F. Dalfovo and M. Modugno, Phys. Rev. A 61, 023605 (2000).

[28] A. L. Gaunt, T. F. Schmidutz, I. Gotlibovych, R. P. Smith, and Z. Hadzibabic, Phys. Rev. Lett. 110, 200406 (2013).

[29] The quantity $\delta N_{n}^{\mathrm{tr}}=\int d t I_{N_{n}}$ differs from the difference in normal atom numbers $\delta N_{n}$ because in each reservoir the normal fraction satisfies $N_{n} / N=\left(T(t) / T_{c}(t)\right)^{3 / 2}$.

[30] E. Lucioni, B. Deissler, L. Tanzi, G. Roati, M. Zaccanti, M. Modugno, M. Larcher, F. Dalfovo, M. Inguscio, and G. Modugno, Phys. Rev. Lett. 106, 230403 (2011). 